\title{
Study of the Penetration and Diffusion Characteristics of Inorganic Solidified Foam in Rock Fractures
}

\author{
Yi Lu, ${ }^{1,2,3}$ Tao Wang, ${ }^{3}$ and Qing Ye \\ ${ }^{1}$ Work Safety Key Lab on Prevention and Control of Gas and Roof Disasters for Southern Coal Mines, \\ Hunan University of Science and Technology, Xiangtan, Hunan 411201, China \\ ${ }^{2}$ Hunan Province Key Laboratory of Safe Mining Techniques of Coal Mines, Hunan University of Science and Technology, \\ Xiangtan, Hunan 411201, China \\ ${ }^{3}$ School of Resource, Environment and Safety Engineering, Hunan University of Science and Technology, \\ Xiangtan, Hunan 411201, China \\ Correspondence should be addressed to Yi Lu; luyijx@163.com
}

Received 5 July 2017; Revised 12 August 2017; Accepted 22 August 2017; Published 28 September 2017

Academic Editor: Marco Rossi

Copyright (C) 2017 Yi Lu et al. This is an open access article distributed under the Creative Commons Attribution License, which permits unrestricted use, distribution, and reproduction in any medium, provided the original work is properly cited.

\begin{abstract}
To explore the penetration and diffusion law in coal and rock fractures when inorganic solidified foam (ISF) is used to prevent coal fire, the penetration experiment was conducted; the results showed that the penetration pressure fluctuates within a certain range and decreases with the diffusion distance. In the $X Y$ plane, the diffusion pattern presents an ellipsoid shape, and the diffusion area becomes increasingly large over time; in the $X Z$ plane, the foam fluid penetration changes from dense to loose in the $X$ direction and it does not undergo downward penetration and diffuses via its own weight in the $Z$ direction; in the $Y Z$ plane, it is loose on the left and dense on the right. The viscosity of ISF was tested and then the time-varying formula was fitted. The formula of the effective diffusion radius for foam fluid diffusing in the fracture channel was determined theoretically. The permeability coefficient and other related parameters were calculated in terms of the penetration pressure and diffusion time of two monitoring points. At last, the prediction formula of effective diffusion distance of foam fluid was verified with the remaining seven monitoring points and all the relative error of monitoring is within $10 \%$.
\end{abstract}

\section{Introduction}

Continuous oxygen is one of the essential conditions for spontaneous combustion of a coal seam $[1,2]$. Mine fire or coal fires are induced by the air leakage passages that provide oxygen for loose coal [3]. However, the fracture channels of the fire area are often complex and crisscross each other, even extending to the high places. In recent years, increasing numbers of scholars began to study foam sealing materials, both at home and abroad, because such materials can diffuse and accumulate in the high fractures and provide stereoscopic covering. The materials of inert gas bubble [4], inhibitor foam [5, 6], gel foam [7-10], three-phase foam [1113], and foamed grout [14-16] are the primary foam materials used for a coal fire. Each of the above-mentioned materials has its own advantages and disadvantages.
To address the disadvantages of the above-mentioned materials, we performed research on ISF fire-fighting technology [17]. ISF can solve the problems of cooling, plugging, insulation, and compressive strength. The fresh state of ISF (foam fluid) can accumulate to a high position as well as cover and cool high temperature coal. Simultaneously, ISF has good insulating ability, thermal stability, and adjustable coagulation time [18]. When it is solidified, ISF has high porosity and compressive strength. The injection of ISF is a very complicated process. To ensure that the foam fluid effectively reaches the coal fire area and seals the fractures, it is necessary to conduct research on the penetration and diffusion laws of the injection process. However, because the injection of ISF is a process with strong concealment, it is difficult to obtain fluid mechanics parameters and collect internal migration images of the foam fluid itself inside the loose coal and rock fractures. 


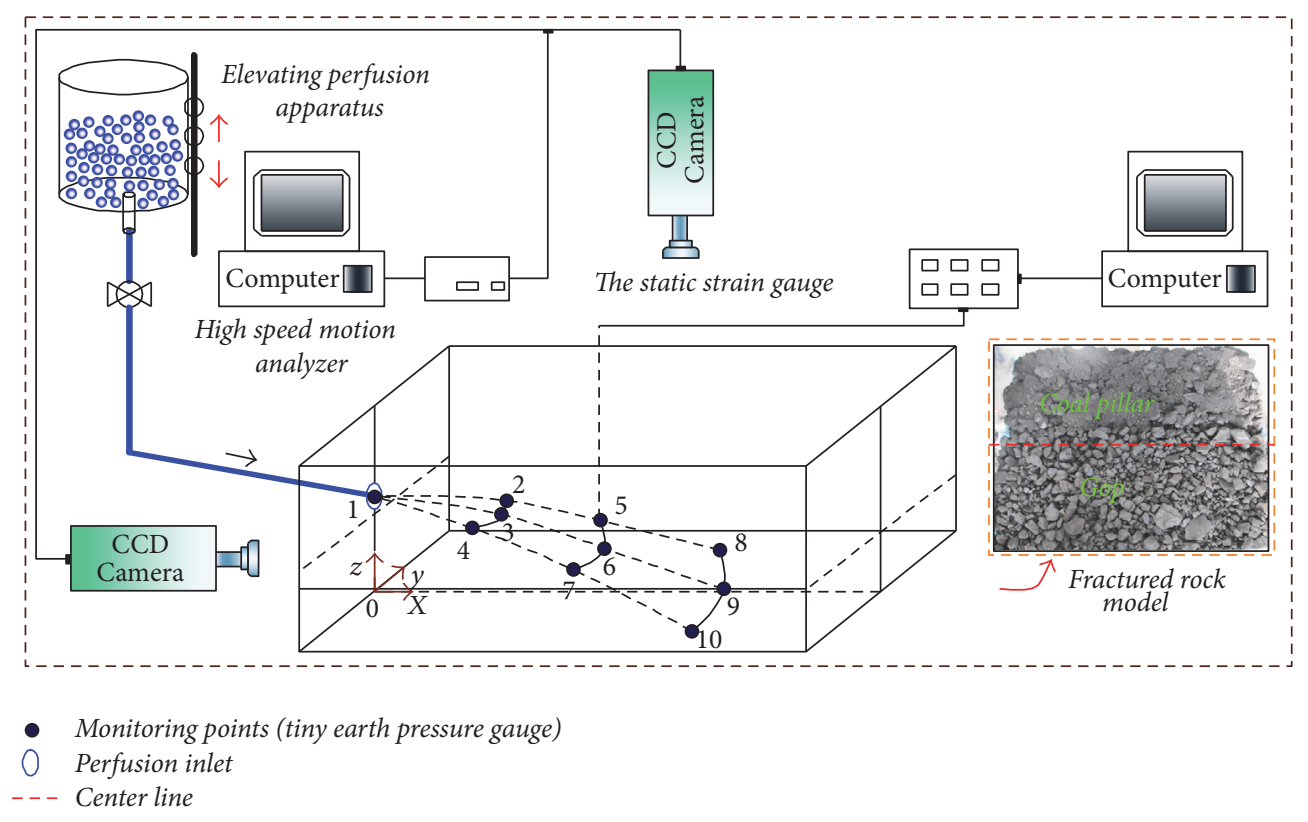

Figure 1: The test system.

In previous studies, a similar, complex model worked out by Gustafson and Stille for predicting grout penetration in real rock was examined by Fujita et al. considering 1D condition $[19,20]$. The latter also reviewed various other grout penetration models developed and used in Japan and elsewhere. Yang et al. investigated the rheological properties of cement grouts with different water cement ratios and their flow in fractures [21]. The present study deals with some of these issues and describes and discusses theoretical grout flow models for predicting flow into plane-parallel fractures of candidate cement grout materials that behave as Bingham fluids under static pressure and as Newton liquids under oscillatory pressure. However, little attention has been paid to the penetration characteristics of foam fluid in the rock fractures [22]. Therefore, its flowing diffusion range, stacking height, and influence factors have not been well understood which causes its blind application in fire controlling. To deal with this problem and provide basis for the design parameters of ISF, this paper puts forward a perfusion experiment of ISF in a fractured rock model that was built according to the site application conditions in Luwa coal mine through actually detecting some of the key parameters of the hydrodynamics of a foam fluid in the fractures, identifying the relationship of various factors in the process of injection, and then summarizing the penetration and diffusion laws of a foam fluid inside the fractures. Combined with the diffusion morphological image of the foam fluid in different directions, the diffusion regularity of the foam fluid is deduced.

\section{Experimental Procedures}

2.1. The Test System. The whole test system (Figure 1) is divided into three parts: elevating perfusion apparatus, fractured rock model, and monitoring system. The fractured rock model consists of a loose coal pillar with the porosity of 0.15 and the surrounding gob with the porosity of 0.35 . The ISF was prepared by the system composed of a foam generator and a self-made mixer. The prepared foam fluid was lifted in an elevating perfusion apparatus, which can cause the foam to flow with a certain pressure head, thereby enabling the fracture rock model to be infused. The main steps of model test are as follows. First, build the similarity model according to the design requirements of filling mediums, and then bury the tiny earth pressure gauges in the process of layering. Second, connect the monitoring equipment; debug the highspeed motion analyser and the static strain gauge. Third, pour the prepared ISF fluid into the fractured rock model using a designed outlet pressure based on the regulating lifting height of the elevating perfusion apparatus. Four, record the realtime diffusion image and the pressure data from the detector, and then analyse and determine the penetration and diffusion laws combined with the viscosity parameters of the ISF fluid. The interface microstructure was investigated using scanning electron microscopy (SEM) (FEI Quanta TM 250 SEM system) with the size of the test specimen being a $10 \mathrm{~mm} \times$ $10 \mathrm{~mm} \times 10 \mathrm{~mm}$ prism.

\subsection{Arrangement of the Testing Points for Penetration Pressure} Monitoring. There are ten tiny earth pressure gauges buried in the fractured rock model. The coordinates of the testing points are listed in Table 1.

In the injection process of ISF, the penetration pressure of the foam fluid in the fracture channels can be monitored by the tiny earth pressure gauges embedded in the fractured rock model. The data collection interval is two seconds. The penetration test ends after all the penetration pressure monitoring data have been collected; the whole experiment takes approximately 15 minutes to complete. The size of the 
TABLE 1: Coordinates of the monitoring points.

\begin{tabular}{lccc}
\hline $\begin{array}{l}\text { Number of the tiny } \\
\text { earth pressure gauges }\end{array}$ & $\begin{array}{c}X \text { coordinate } \\
(\mathrm{mm})\end{array}$ & $\begin{array}{c}Y \text { coordinate } \\
(\mathrm{mm})\end{array}$ & $\begin{array}{c}Z \text { coordinate } \\
(\mathrm{mm})\end{array}$ \\
\hline $1 \#$ & 0 & 0 & 300 \\
$2 \#$ & 232 & 93 & 250 \\
$3 \#$ & 250 & 0 & 250 \\
$4 \#$ & 232 & -93 & 250 \\
$5 \#$ & 464 & 186 & 200 \\
$6 \#$ & 500 & 0 & 200 \\
$7 \#$ & 464 & -186 & 200 \\
$8 \#$ & 696 & 279 & 100 \\
9\# & 750 & 0 & 100 \\
$10 \#$ & 696 & -279 & 100 \\
\hline
\end{tabular}

TABLE 2: Offered parameters of the $0.1 \mathrm{MPa}$ tiny earth pressure gauges.

\begin{tabular}{lcc}
\hline $\begin{array}{l}\text { Number of the tiny } \\
\text { earth pressure gauges }\end{array}$ & \multicolumn{2}{c}{ Offered parameters } \\
\hline $1 \#$ & -0.80875 & $b$ \\
$2 \#$ & -1.58700 & 0.06873 \\
$3 \#$ & -1.44946 & 0.05042 \\
$4 \#$ & -1.22361 & 0.05691 \\
$5 \#$ & -0.92195 & 0.05934 \\
$6 \#$ & -1.43612 & 0.06540 \\
$7 \#$ & -1.02616 & 0.06260 \\
$8 \#$ & -0.34060 & 0.06676 \\
$9 \#$ & -1.11560 & 0.08350 \\
$10 \#$ & -1.02340 & 0.06587 \\
\hline
\end{tabular}

tiny earth pressure gauge is $15 \mathrm{~mm}$ (diameter) $\times 6.4 \mathrm{~mm}$ (thickness), maximum measurement value is $0.1 \mathrm{Mpa}$, and the accuracy is $\pm 1.0 \%$ F.S. The computation formula between fluid penetration pressure and dependent variable is as follows:

$$
P=a+b \varepsilon \text {. }
$$

In formula (1), $P$ is the value of penetration pressure, $\mathrm{kPa}$; $\varepsilon$ is the dependent variable, $\times 10^{-6} \varepsilon ; a$ and $b$ are the offered parameters. After calibrating the foam fluid, the determined offered parameters are listed in Table 2.

\section{The Test Result Analysis}

3.1. The Penetration Pressure Analysis. According to the offered parameters of the $0.1 \mathrm{MPa}$ tiny earth pressure gauges shown in Table 2, the penetration pressure of foam fluid can be calculated. The pressure values over time are shown in Figures 2 and 3.

From Figures 2 and 3, overall, the 1\# 10\# monitoring point data fluctuates up and down within a certain range.

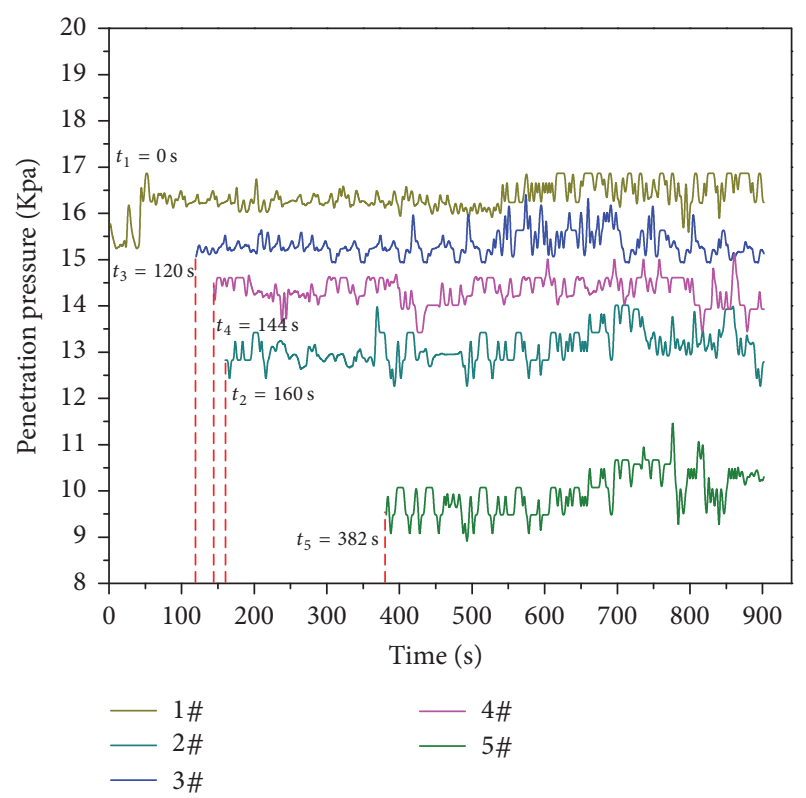

FIGURE 2: The change of penetration pressure over time from monitoring points $1 \#-5 \#$.

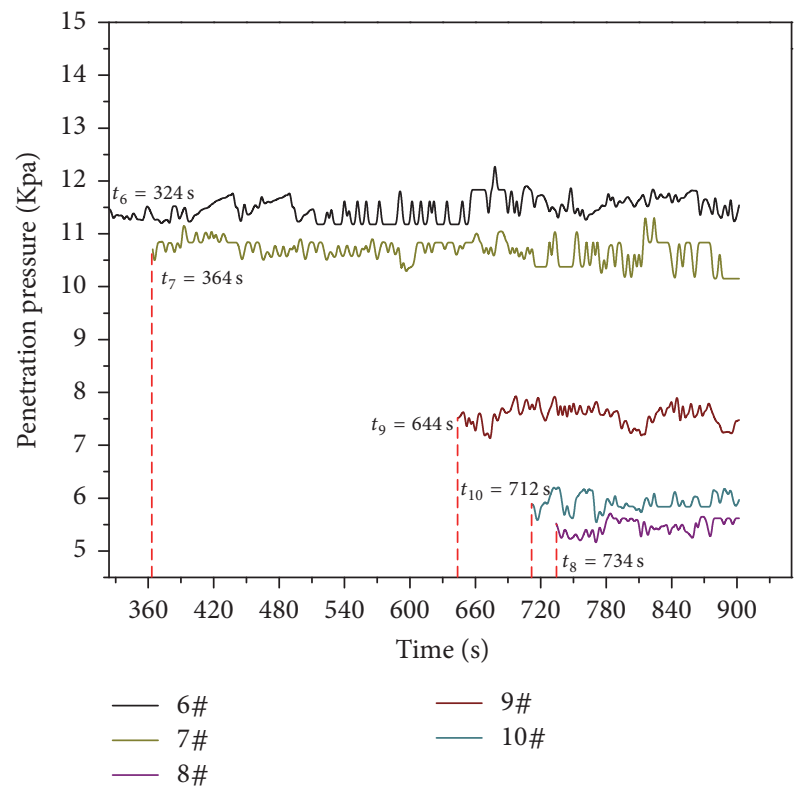

FIGURE 3: The change of penetration pressure over time from monitoring points $6 \#-10 \#$.

In addition to the $1 \#$ monitoring point (the injection inlet), overall, the monitoring points are divided into three groups: the first group contains the $2 \#, 3 \#$, and $4 \#$ monitoring points, the second group consists of the 5\#,6\#, and 7\# monitoring points, and the third group is composed of the 8\#, 9\#, and $10 \#$ monitoring points. With the increase of diffusion distance, the penetration pressure decreases, in agreement with the traditional grouting penetration diffusion law. The penetration pressure of the $1 \#$ monitoring point fluctuates 
with the value of $16.37 \mathrm{kPa}$, which was obtained from the beginning. The first group of $2 \#, 3 \#$, and $4 \#$ monitoring points was buried separately within the loose coal pillar, the centre line, and the surrounding gob. The penetration diffusion distance of the 2\#, 3\#, and 4\# monitoring points is $255 \mathrm{~mm}$, $250 \mathrm{~mm}$, and $255 \mathrm{~mm}$, respectively. The $3 \#$ monitoring point first collects the penetration pressure at the moment of $120 \mathrm{~s}$, and the $2 \#$ monitoring point and $4 \#$ monitoring point collect the penetration pressure at the moment of $160 \mathrm{~s}$ and $144 \mathrm{~s}$, respectively. This phenomenon occurs primarily because the porosity of loose coal pillar is smaller than the surrounding gob, and the frictional resistance loss in the fracture channel is high. As a result, under the condition of the same $255 \mathrm{~mm}$ diffusion distance, it takes a longer time for the foam fluid to diffuse to the $2 \#$ monitoring point. In addition, the average penetration pressure of the $2 \#$ monitoring point is $13.13 \mathrm{kPa}$, which is smaller than the average penetration pressure of the $3 \#$ monitoring point $(14.97 \mathrm{kPa})$ and the average penetration pressure of the $4 \#$ monitoring point $(14.36 \mathrm{kPa})$. The set of $5 \#$, 6\#, and 7\# monitoring points is buried in the same manner as the first group of $2 \#, 3 \#$, and $4 \#$ monitoring points. The 5\#, 6\#, and 7\# monitoring points are located along the centre line, the loose coal pillar, and the surrounding gob, and the corresponding diffusion distance is $510 \mathrm{~mm}$, $500 \mathrm{~mm}$, and $510 \mathrm{~mm}$, respectively. As the first group of data, the 6\# monitoring point in the centre line first collected the penetration pressure at the moment of $324 \mathrm{~s}$, with the average penetration pressure value of $11.94 \mathrm{kPa}$. Next is the 7\# monitoring point arranged in the goaf at $364 \mathrm{~s}$, with the average penetration pressure value of $10.72 \mathrm{kPa}$. Finally, the 5\# monitoring point in the loose coal pillar collects the penetration pressure at the time of $382 \mathrm{~s}$ : the average value of penetration pressure is $10.05 \mathrm{kPa}$.

The penetration diffusion time and the penetration pressure relationship of the third group of monitoring points $8 \#, 9 \#$, and 10\# are similar to those of the first two groups. The 8\#, 9\#, and 10\# monitoring points' penetration diffusion distances are $776 \mathrm{~mm}, 750 \mathrm{~mm}$, and $776 \mathrm{~mm}$, respectively. The 9\# monitoring point in the centre line first detected the penetration pressure with the value of $7.63 \mathrm{kPa}$ at the moment of $644 \mathrm{~s}$. Subsequently, the 10\# monitoring point arranged in the goaf detected the penetration pressure at $712 \mathrm{~s}$ : the average penetration pressure value is $5.98 \mathrm{kPa}$. Finally, the $5 \#$ monitoring points in the loose coal pillar detected the penetration pressure at $734 \mathrm{~s}$ : the average value is $5.52 \mathrm{kPa}$. Although the penetration diffusion distance difference of the three groups is approximately $250 \mathrm{~mm}$, the penetration diffusion time and the penetration pressure average value difference of the three groups are increasing because the foam fluid diffuses in loose coal and rock fracture channel with energy loss, thereby causing the speed to be increasingly low. Therefore, under the same penetration diffusion distance, the time required for the latter half of the distance becomes increasingly long, and the pressure difference becomes increasingly high.

3.2. Penetration Diffusion Image Analysis. Here, the data from the monitoring points buried within the model is analysed. To more vividly illustrate the diffusion form of the foam fluid, a high-speed motion analyser was adopted in the whole experiment; the $X-Y$ plane of the foam fluid penetration diffusion pattern is shown in Figure 4.

From Figure 4, with the increase of diffusion time, the diffusion form of the foam fluid in the $X-Y$ plane becomes ellipsoidal. The diffusion velocity in the loose coal pillar and the surrounding gob is not the same, as the diffusion area of the foam fluid in the surrounding gob is larger than that of the loose coal pillar. This difference is primarily because the fracture porosity of the loose coal pillar is small, causing the foam fluid to experience greater free diffusion resistance. From the diffusion area size at different moments, the diffusion area is increasing with time, but the diffusion area of growth becomes smaller. This behaviour occurs primarily because the foam fluid is a time-varying Bingham fluid, resulting in the viscosity increasing from $4360 \mathrm{MPa} \cdot \mathrm{s}$ at $60 \mathrm{~s}$ to $4451 \mathrm{MPa} \cdot \mathrm{s}$ at $360 \mathrm{~s}$, causing the foam fluid flows to slow. Simultaneously, with the increase of the diffusion range, the penetration pressure drop of the foam fluid in the complex fracture network is gradually reduced and the front diffusion of foam fluid slows. In addition, the base material in the pore wall of the foam fluid undergoes hydration and condensation reactions $[23,24]$, thus causing the stability of the bubble to enhance that in turn makes it difficult to change the shape of the structure. In the SEM image obtained from the bubble wall (Figure 5), we can observe that the rod-shaped ettringite crystals fill the capillary pores. Surface products such as C$\mathrm{S}-\mathrm{H}$ gel can be observed as the major ISF microstructure component. $\mathrm{CH}$ as a pore product with a polycrystalline shape is another dominant cement hydration product. The phenomenon results in a large quantity of bubbles uniting into a group. The bubbles in the group flow as a whole and block each other. The fluidity of the foam fluid reduces gradually. Figure 6 shows the condensation effect of foam fluid in a coal and rock fracture surface after 3 hours. The figure reveals that the foam fluid can commendably diffuse and cover the fracture surface, and the foam can form a layer of a certain thickness, effectively isolating the coal and oxygen compounds.

To perform in-depth analysis of the internal penetration diffusion of the ISF in the fractured rock model, the $X Z$ and $Y Z$ sections were recorded by the CCD camera, as shown in Figures 7 and 8, respectively.

From Figure 7, in the $X Z$ penetration section, with the increase of the penetration diffusion distance, the foam fluid penetration in the fracture channel changes from dense to loose in the $X$ direction, mainly because the penetration pressure and the velocity of the foam fluid are high near the perfusion inlet, thereby enabling the foam to diffuse fully. However, the penetration pressure loss of the foam fluid is higher when the position is further away from the perfusion inlet. From the $Z$ direction, the fracture penetration is fuller at the same $Z$ height of the perfusion inlet; the next place is above the plane of the perfusion inlet, and the penetration diffusion effect is the worst in the sections under the height of the perfusion inlet. It indicates that the penetration diffusion ability of the foam fluid is stronger in its initial direction of velocity vector than the direction of the vector upward or downward. Upward penetration is fuller than the downward penetration because the peripheral interface cracks in the 

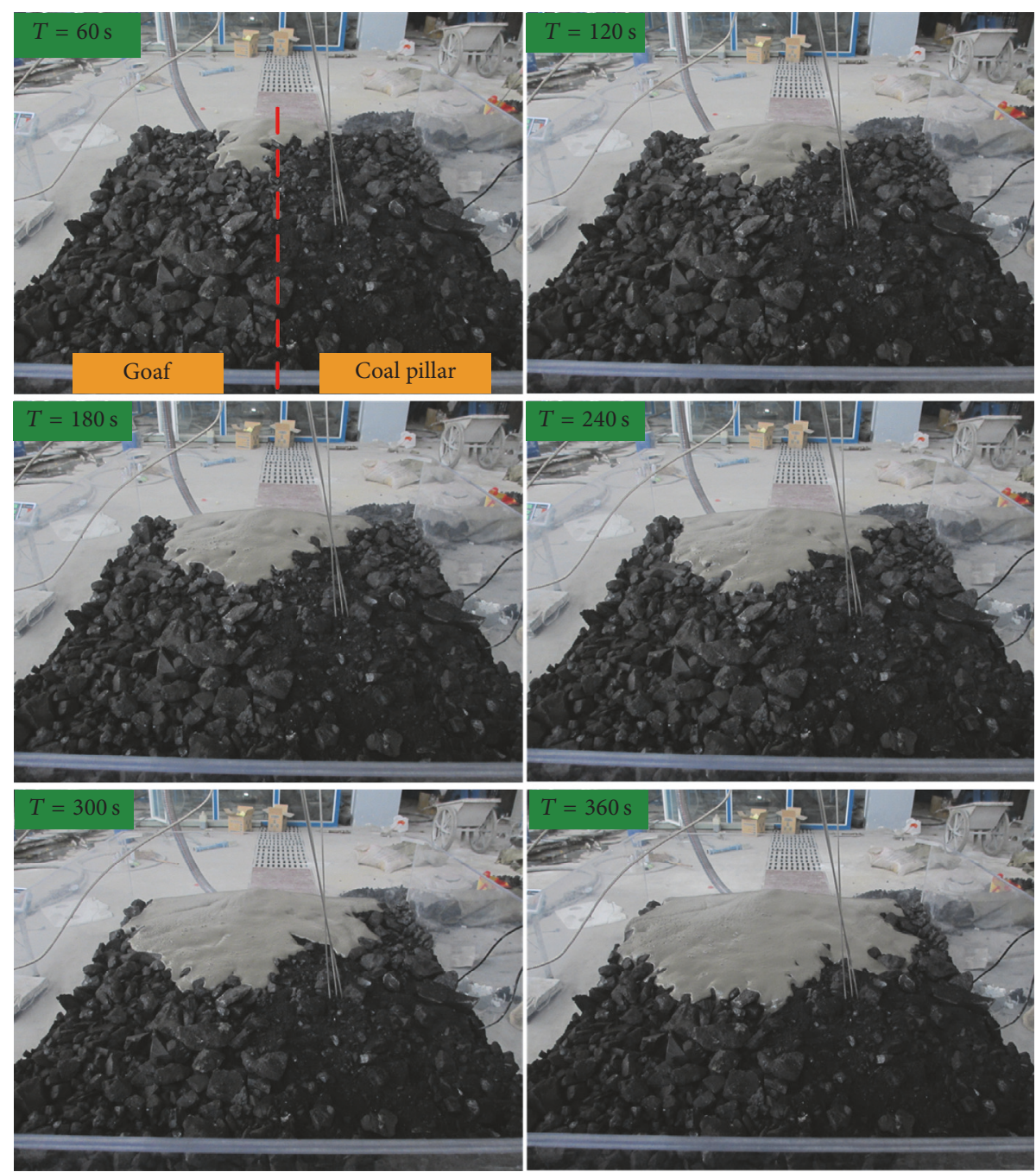

Figure 4: The penetration and diffusion form of the foam fluid in the $X-Y$ plane.

process of planar penetration channels are open and the crack channel resistance is low, indicating that the foam fluid does not act in the same manner as normal cement slurry. This difference in downward penetration mainly occurs because the density of foam fluid is smaller than that of ordinary cement, with the value being approximately 1/5 the density of a common slurry. As a result, the gravitational effect on the foam fluid is not obvious. Another primary factor is that the foam fluid is composed of bubbles; as a result, adhesion of cement and fly ash particles occurs in the bubble hole wall or the particles exist in a foam liquid membrane to form a skeleton, thereby preventing gravity settling of the particles. The size of a single bubble is approximately 400 microns, and each bubble links together, thereby causing the bubble size to be greater than a single general cement or fly ash particle in the slurry.

Form the penetration and diffusion in the $Y Z$ section shown in Figure 8, the left is loose and the right is dense in the $Y$ direction, largely because the left part is the loose coal pillar model with the porosity of 0.15 and the right part is the surrounding gob model with the porosity of 0.35 . As the perfusion inlet is in the cross section, the foam fluid will be more easily diffuse into the right side with large porosity. In the $Z$ direction, the distribution of penetration and diffusion is uniform, except the top with the surface penetration, mainly because perfusion inlet of foam fluid is in the $X$ direction, which is vertical to the $Y Z$ section. The penetration and diffusion of foam fluid in this $Y Z$ section occurred when the fracture has been blocked by the $X$ direction penetration. The up and down diffusion process was mainly affected by the width of fracture in the $Z$ direction. Thus, in the $Y Z$ plane, the penetration and diffusion results show that the left part is loose and right part is dense.

3.3. Penetration and Diffusion Law. In the grouting engineering field, there is a large difference for different flow patterns of slurry diffuse in the geotechnical engineering [25-27]. To meet the requirements of engineering, reasonable grouting parameters for different slurry must be determined. However, the determination of the grouting parameters definitely 


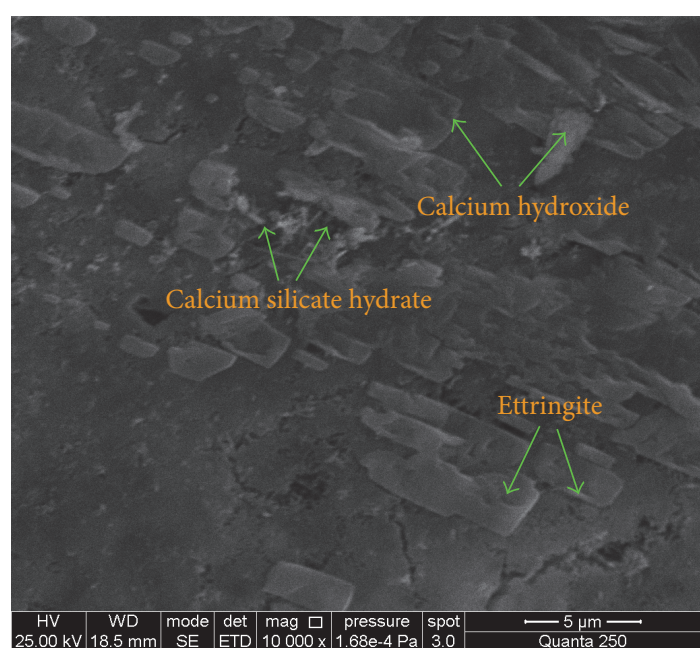

FIGURE 5: SEM image obtained from the bubble wall.

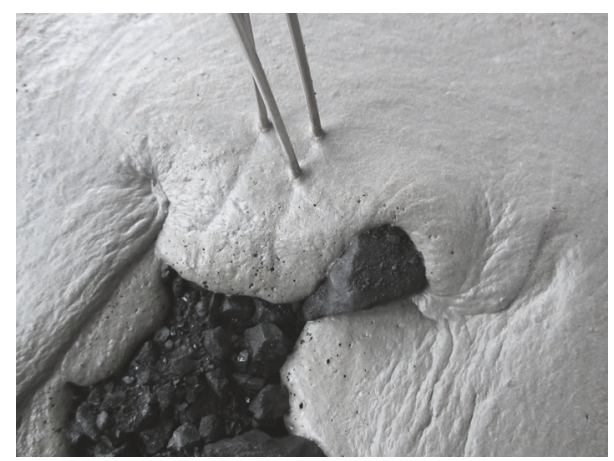

FIGURE 6: Condensation effect of a fluid penetration diffusion front in the coal and rock surface after 3 hours.

requires guidance from grouting theory. The relatively mature diffusion grouting theory is based on the spherical, cylindrical, and sleeve valve pipe models [28-31]. The effective diffusion radius formula is only applicable to a Newtonian fluid. However, the inorganic solidified foam is a Bingham fluid, which is a non-Newtonian fluid; as a result, such foam cannot be modelled by the above formulas. Therefore, in this paper, the diffusion theory of the ISF in porous media with fracture channels is derived. As the ISF fluid diffuses completely into the fractures channel, we assume that it is a whole unit mass of foam fluid that reaches a certain position along a fracture channel. Therefore, we can draw lessons from the model of a Bingham fluid in pipe penetration [32], as shown in Figure 9.

Assuming that the fracture radius is $r_{0}$, a foam fluid unit is taken from the centre line position of the fracture channel; its length is $d l$ and its radius is $r$. The pressure of the left and right ends of the foam fluid unit $d l$ is $p+d p$ and $p$, respectively. The differential pressure is $d p$. The shear stress around the foam fluid unit surface by the left direction (in the opposite direction of flow velocity) is $\tau$. Thus, we obtain the balance

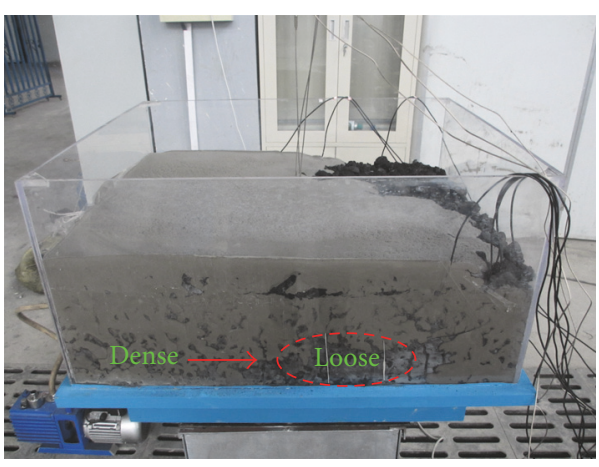

Figure 7: Penetration and diffusion form of the foam fluid in the $X-Z$ section.

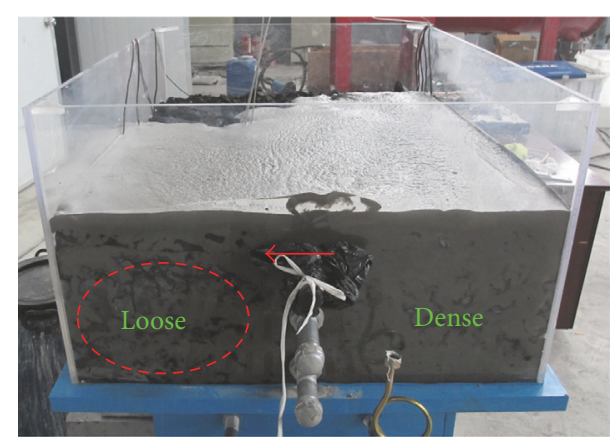

Figure 8: Penetration and diffusion form of the foam fluid in the $Y-Z$ section.

equation for flow stress of a foam fluid unit as follows:

$$
\pi r^{2} d p=-2 \pi r \tau d l
$$

In the region of $0 \leq r \leq r_{p}$, the foam fluid column is stationary relative to the adjacent layer fluid. The foam fluid moves in a piston as a whole, and the movement velocity is $v=v_{p}$. In the region of $r_{p} \leq r \leq r_{0}$, the foam fluid column is moving relative to the adjacent layer fluid. So the following equation can be obtained:

$$
r_{p}=\frac{-2 \tau_{s} \cdot d l}{d p} .
$$

The basic rheological equation of a Bingham fluid slurry [33] can be expressed as

$$
\tau=\tau_{s}+\eta_{p} \gamma
$$

Based on (2) and (4), the following can be obtained:

$$
\gamma=-\frac{d v}{d r}=\frac{\left(\tau-\tau_{s}\right)}{\eta_{p}}=-\frac{1}{\eta_{p}} \cdot\left(\frac{r}{2} \cdot \frac{d p}{d l}+\tau_{s}\right) .
$$

Consider boundary conditions $r=r_{0}, v=0$ and then calculate integral for both sides of (5).

$$
\begin{aligned}
\int_{v}^{v_{0}} d v & =\int_{r}^{r_{0}}\left[\frac{1}{\eta_{p}} \cdot\left(\frac{r}{2} \cdot \frac{d p}{d l}+\tau_{s}\right)\right] \cdot d r \Longrightarrow \\
v & =-\frac{1}{\eta_{p}}\left[\frac{1}{4} \frac{d p}{d l}\left(r_{0}^{2}-r^{2}\right)+\tau_{s}\left(r_{0}-r\right)\right] .
\end{aligned}
$$




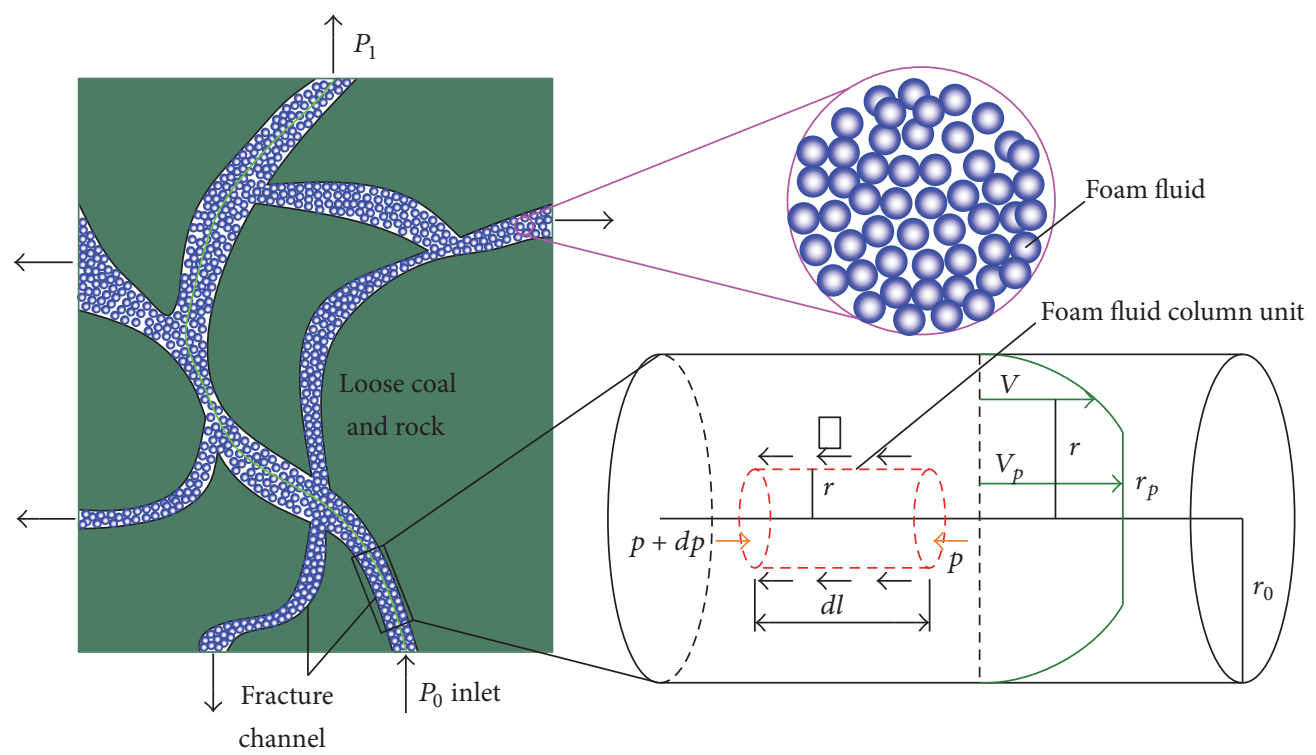

FIGURE 9: Schematic of the column flow of a unit of foam fluid in a fracture channel.

For $0 \leq r \leq r_{p}$

$$
v_{p}=-\frac{1}{\eta_{p}}\left[\frac{1}{4} \frac{d p}{d l}\left(r_{0}^{2}-r_{p}^{2}\right)+\tau_{s}\left(r_{0}-r_{p}\right)\right] .
$$

Therefore, the flow of fracture channel per unit time is the sum of the shear zone and the piston area, namely,

$$
\begin{aligned}
Q_{1}= & \pi r_{p}^{2} v_{p}+\int_{r_{p}}^{r_{0}} 2 \pi r v d v \\
= & -\frac{4 \pi \tau_{s}^{2}}{(d p / d l)^{2}} \cdot \frac{1}{\eta_{p}} \cdot\left[\frac{1}{4} \frac{d p}{d l}\left(r_{0}^{2}-r_{p}^{2}\right)+\tau_{s}\left(r_{0}-r_{p}\right)\right] \\
& +\int_{r_{p}}^{r_{0}} 2 \pi \cdot \frac{r}{\eta_{p}} \\
& \cdot\left[\frac{1}{4} \frac{d p}{d l} r_{0}^{2}+\tau_{s} r_{0}-\frac{1}{4} \frac{d p}{d l} r_{p}^{2}-\tau_{s} r_{p}\right] d r \\
= & \frac{2}{3} \frac{\pi \tau_{s}^{4}}{\eta_{p}(d p / d l)}-\frac{\pi \tau_{s} r_{0}^{3}}{3 \eta_{p}}-\frac{\pi \tau_{s} r_{0}^{4}}{8 \eta_{p}} \cdot \frac{d p}{d l} .
\end{aligned}
$$

The average flow velocity of the section of fracture channel can be expressed as

$$
V=\frac{K}{\beta}\left(-\frac{d p}{d l}\right)\left[1-\frac{4}{3}\left(\frac{\lambda}{-d p / d l}\right)+\frac{1}{3}\left(\frac{\lambda}{-d p / d l}\right)^{4}\right]
$$

We make three assumptions for the penetration and diffusion of foam fluid in the fracture channel: (1) the coal pillar and circumferential crack area are all homogeneous and isotropic; (2) the foam fluid is a Bingham fluid; and (3) the spreading form of the foam fluid is spherical diffusion. In the injection process of a foam fluid, the injection content, $Q$, satisfies

$$
Q=V A t
$$

In (10), $A$ is a sphere when the foam fluid diffuses in the fracture channel, $A=4 \pi l^{2} ; t$ is the grouting time; $-d p / d l$ is much larger than $\lambda$ in the injection process. Thus, (9) can be simplified as

$$
V=\frac{K}{\beta}\left(-\frac{d p}{d l}\right)\left[1-\frac{4}{3}\left(\frac{\lambda}{-d p / d l}\right)\right] .
$$

Combining (2) and (4) and then calculating the integral for both sides of the equation, the following is obtained:

$$
p=\frac{Q \beta}{4 \pi t K l}-\frac{4}{3} \lambda l+C
$$

Considering the boundary conditions of injecting foam fluid (i.e., for $p=p_{0}, l=l_{0}$, and for $p=p_{1}, l=l_{1}$ ), the following is obtained:

$$
\Delta p=\frac{\phi \beta}{3 t K l_{0}} l_{1}^{3}-\frac{\phi \beta}{3 t K} l_{1}^{2}+\frac{4}{3} \lambda l_{1}-\frac{4}{3} \lambda l_{0} .
$$

Equation (13) is the calculation formula of the effective diffusion radius for foam fluid diffusing in the fracture channel, where $\Delta p$ is the penetration pressure difference between two monitoring points in the fracture channel; $\phi$ is the porosity: the porosity of coal pillar in the test model is 0.15 and the porosity of the goaf is $0.35 ; \beta$ is the viscosity ratio of foam fluid and water; $t$ is the grouting time; $K$ is the permeability coefficient; $\lambda$ is the ratio of two times the static shear force and the fracture channel radius; $l_{0}$ is the radius of injection inlet with the value of $15 \mathrm{~mm}$; $l_{1}$ is the penetration and diffusion distance.

Penetration time is a major issue in the grouting of rock fractures. The foam fluid state will be calculated as a time function, making a distinction between flow rate control and pressure control. The indoor environment temperature 
TABLE 3: Penetration pressure and diffusion time of the monitoring points.

\begin{tabular}{|c|c|c|c|c|c|c|c|c|c|c|}
\hline Monitoring points & $1 \#$ & 2\# & $3 \#$ & $4 \#$ & $5 \#$ & $6 \#$ & 7\# & $8 \#$ & 9\# & $10 \#$ \\
\hline $\begin{array}{l}\text { Average penetration } \\
\text { pressure }(\mathrm{kPa})\end{array}$ & 16.47 & 13.13 & 14.97 & 14.36 & 10.05 & 11.94 & 10.72 & 5.52 & 7.63 & 5.98 \\
\hline Diffusion time (s) & 0 & 160 & 120 & 144 & 382 & 324 & 364 & 734 & 644 & 712 \\
\hline $\begin{array}{l}\text { Differential pressure } \\
\text { with } 1 \#(\mathrm{kPa})\end{array}$ & 0 & 3.24 & 1.5 & 2.01 & 6.32 & 4.53 & 5.65 & 10.85 & 8.74 & 10.39 \\
\hline $\begin{array}{l}\text { Viscosity of ISF fluid } \\
(\mathrm{Pa} \cdot \mathrm{s})\end{array}$ & 4.360 & 4.370 & 4.360 & 4.365 & 4.451 & 4.448 & 4.454 & 4.557 & 4.523 & 4.548 \\
\hline Diffusion distance $(\mathrm{mm})$ & 0 & 255 & 250 & 255 & 510 & 500 & 510 & 776 & 750 & 776 \\
\hline Porosity & 0.35 & 0.15 & 0.35 & 0.35 & 0.15 & 0.35 & 0.35 & 0.15 & 0.35 & 0.35 \\
\hline
\end{tabular}

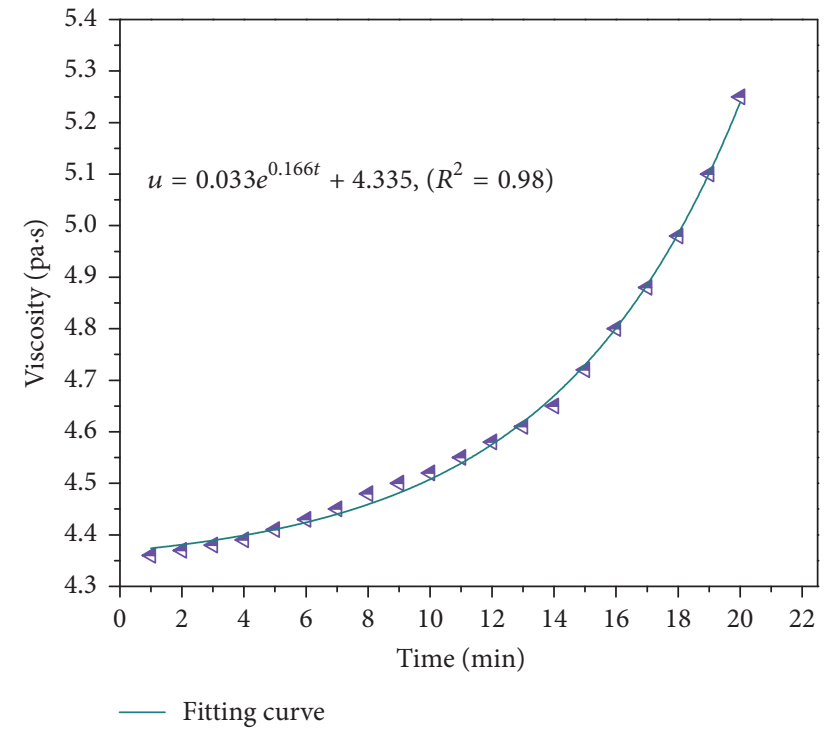

FIGURE 10: Foam fluid viscosity varies over time.

is $10^{\circ} \mathrm{C}$, and the viscosity of the water is $1.3077 \mathrm{MPa} \cdot \mathrm{s}$, based on the viscosity of the water table. The viscosity of the ISF fluid was measured using a NDJ-5s rotary viscometer, setting the rotor number to 2 and the rotor speed to 6 . The viscosity varies with time, as shown in Figure 10.

The change of viscosity over time was fitted in Figure 10; the fitting function obtained is given by

$$
u=a e^{b t}+c
$$

where $a=0.033, b=0.166$, and $c=4.335$; the correlation coefficient is $R^{2}=0.98$. As a result, we can obtain the viscosity ratio of foam fluid and water using

$$
\beta=\frac{u_{\mathrm{ISF}}}{u_{W}}=\frac{a e^{b t}+c}{u_{W}}=\frac{0.033 e^{0.166 t}+4.335}{1.3077 \times 10^{-3}} .
$$

From Figures 2 and 3, the average penetration pressure and the diffuse time of monitoring points $1 \#-10 \#$ are listed in Table 3.
Based on (13), the relationship among the differential pressure of the $1 \#$ monitoring point, the diffusion time, and the diffusion distance can be expressed as

$$
\begin{aligned}
& \Delta p_{13}=\frac{\phi_{3} \beta_{3}}{3 t_{3} K l_{0}} l_{13}^{3}-\frac{\phi_{3} \beta_{3}}{3 t_{3} K} l_{13}^{2}+\frac{4}{3} \lambda l_{13}-\frac{4}{3} \lambda l_{0}, \\
& \Delta p_{16}=\frac{\phi_{6} \beta_{6}}{3 t_{6} K l_{0}} l_{16}^{3}-\frac{\phi_{6} \beta_{6}}{3 t_{6} K} l_{16}^{2}+\frac{4}{3} \lambda l_{16}-\frac{4}{3} \lambda l_{0} .
\end{aligned}
$$

We substituted the related parameters in the Table 3 into (16) to obtain

$$
\begin{gathered}
1500=\frac{1.3665}{K}+0.3133 \lambda, \\
4530=\frac{9.8377}{K}+0.6467 \lambda, \\
\Downarrow \\
K=4.894 \times 10^{-3}(\mathrm{~m} / \mathrm{s}), \\
\lambda=3.897 \times 10^{3}(\mathrm{~Pa} / \mathrm{m}) .
\end{gathered}
$$

To further amend the formula of effective diffusion distance of foam fluid, the diffusion time and diffusion distance of the remaining monitoring points $2 \#, 4 \#, 5 \#, 7 \#$, $8 \#, 9 \#$, and 10\# were substituted into the formula, and then the predictive values were calculated. The comparison of the predicted values and the test values is provided in Table 4.

From Table 4, the predicted values were overall in accord with the experimental results. The predicted values were all less than the experimental results; this difference occurs mainly because the foam fluid impacts the tiny earth pressure gauges and causes it to be slightly mobile. As a result, the follow-up monitoring data may contain some errors. However, because all the relative error of monitoring is within $10 \%$, the prediction formula is reasonable. Thus, we can obtain the relations of the diffusion distance, penetration pressure difference, and diffusion time given by (18). Based on it, it can be obtained that the penetration pressure difference between two monitoring points $(\Delta p)$ was affected by the porosity $(\phi)$ and the penetration and diffusion distance $\left(l_{1}\right)$. In Figure 7, the porosity of the model is the same. With 
TABLE 4: Comparison of the predicted values and the test values.

\begin{tabular}{lccccccc}
\hline Monitoring point & 2\# & $4 \#$ & $5 \#$ & 7\# & 8\# & 9\# & 10\# \\
\hline Predicted value $(\mathrm{kPa})$ & 2.94 & 1.92 & 6.05 & 5.29 & 9.93 & 7.96 & 9.51 \\
Test value $(\mathrm{kPa})$ & 3.24 & 2.01 & 6.32 & 5.65 & 10.85 & 8.74 & 10.39 \\
Relative error $(\%)$ & 9.25 & 4.48 & 4.27 & 6.37 & 8.47 & 8.92 \\
\hline
\end{tabular}

the increase of the penetration and diffusion distance, the penetration pressure near the perfusion inlet is higher than the position which is further away from the perfusion inlet. For the same penetration and diffusion distance, the greater the porosity is, the smaller the seepage pressure difference is, and the greater the seepage pressure is, which can prove the diffusion morphology in Figure 8.

$$
\begin{aligned}
\Delta p= & \frac{\phi\left(\left(a e^{b t}+c\right) / u_{W}\right)}{3 t K l_{0}} l^{3}-\frac{\phi\left(\left(a e^{b t}+c\right) / u_{W}\right)}{3 t K} l^{2} \\
& +\frac{4}{3} \lambda l-\frac{4}{3} \lambda l_{0} .
\end{aligned}
$$

\section{Conclusions}

A similar fractured rock model was built according to the coal pillar and the surrounding gob. The penetration pressure, image, and law in the complex fracture were investigated. Some conclusions can be drawn as follows.

(1) The pressure fluctuated up and down within a certain range overall. With the increase of diffusion distance, the penetration pressure decreased, consistent with the traditional grouting penetration diffusion law. Affected by the porosity of filling medium, a longer time is required to monitor the penetration pressure for the loose coal pillar than the gob, and the value of penetration pressure in loose coal pillar was lower than that of the gob at the same diffusion distance.

(2) The diffusion form of the foam fluid in the $X-Y$ plane is ellipsoidal, with the diffusion area increasing with time, although the diffusion area of growth becomes smaller with time; in the $X Z$ section, the foam fluid diffusion in the fracture channel is from dense to loose in the $X$ direction, and, from the $Z$ direction, the fracture penetration is fuller at the same $Z$ height of the injection inlet; in the $Y Z$ section, the left part is loose and the right part is dense in the $Y$ direction, and the distribution of penetration and diffusion is uniform, except the top, with surface penetration.

(3) The viscosity of the ISF fluid was measured by a NDJ$5 s$ rotary viscometer. The change of viscosity over time was fitted to obtain the fitting function. Based on the formula of Bingham fluid penetration in a pipe, the formula of the diffusion radius of the ISF was determined theoretically and then compared and verified in terms of the pressure data from the monitoring point.

\section{Conflicts of Interest}

The authors declare that there are no conflicts of interest regarding the publication of this paper.

\section{Acknowledgments}

This work was supported by the National Natural Science Foundation of China (51604110, 51774135, 51504093, and 51374003), Provincial Natural Science of Hunan (2017JJ3074), China Postdoctoral Science Foundation (2017M612558), and Research Project of Hunan Provincial Education Department (17C0641).

\section{References}

[1] Y. Tang, "Inhibition of low-temperature oxidation of bituminous coal using a novel phase-transition aerosol," Energy \& Fuels, vol. 30, pp. 9303-9309, 2016.

[2] J. Pandey, N. K. Mohalik, R. K. Mishra, A. Khalkho, D. Kumar, and V. K. Singh, "Investigation of the Role of Fire Retardants in Preventing Spontaneous Heating of Coal and Controlling Coal Mine Fires," Fire Technology, vol. 51, no. 2, pp. 227-245, 2015.

[3] T. Xia, X. Wang, F. Zhou, J. Kang, J. Liu, and F. Gao, "Evolution of coal self-heating processes in longwall gob areas," International Journal of Heat and Mass Transfer, vol. 86, pp. 861-868, 2015.

[4] H.-Z. Yu, R. Kasiski, and M. Daelhousen, "Characterization of Twin-Fluid (Water Mist and Inert Gas) Fire Extinguishing Systems by Testing and Modeling," Fire Technology, vol. 51, no. 4, pp. 923-950, 2014.

[5] W. Lu, "The high inhibitor bubble for prevention and control of coal spontaneous combustion," Coal Science and Technology, vol. 46, pp. 41-44, 2008.

[6] J. Deng, Y. Xiao, Q. Li, J. Lu, and H. Wen, "Experimental studies of spontaneous combustion and anaerobic cooling of coal," Fuel, vol. 157, pp. 261-269, 2015.

[7] X. W. Ren, F. Z. Wang, Q. Guo, Z. B. Zuo, and Q. S. Fang, "Application of Foam-gel Technique to Control CO Exposure Generated during Spontaneous Combustion of Coal in Coal Mines," Journal of Occupational and Environmental Hygiene, vol. 12, no. 11, pp. D239-D245, 2015.

[8] Y. Lu, "Laboratory study on the rising temperature of spontaneous combustion in coal stockpiles and a paste foam suppression technique," Energy \& Fuels, vol. 31, pp. 7290-7298, 2017.

[9] X. Qi, D. Wang, H. Xin, and X. Zhong, "Environmental hazards of coal fire and their prevention in China," Environmental Engineering and Management Journal, vol. 12, no. 10, pp. 19151919, 2013.

[10] L. Zhang, B. Qin, B. Shi, and K. Wen, "Formation mechanism of foamed gel for controlling the coal spontaneous combustion," Combustion Science and Technology, vol. 188, no. 1, pp. 132-143, 2016.

[11] B. Qin, Y. Lu, Y. Li, and D. Wang, "Aqueous three-phase foam supported by fly ash for coal spontaneous combustion prevention and control," Advanced Powder Technology, vol. 25, no. 5, pp. 1527-1533, 2014. 
[12] Z. Shao, D. Wang, Y. Wang, X. Zhong, X. Tang, and X. Hu, "Controlling coal fires using the three-phase foam and water mist techniques in the Anjialing Open Pit Mine, China," Natural Hazards, vol. 75, no. 2, pp. 1833-1852, 2015.

[13] F. Zhou, W. Ren, D. Wang, T. Song, X. Li, and Y. Zhang, "Application of three-phase foam to fight an extraordinarily serious coal mine fire," International Journal of Coal Geology, vol. 67, no. 1-2, pp. 95-100, 2006.

[14] J. J. Feiler, G. J. Colaizzi, and C. Carder, "Foamed grout controls underground coal-mine fire," Mining Engineering, vol. 52, pp. 58-62, 2000.

[15] G. J. Colaizzi, "Prevention, control and/or extinguishment of coal seam fires using cellular grout," International Journal of Coal Geology, vol. 59, no. 1-2, pp. 75-81, 2004.

[16] X. Lu, D. Wang, C. Zhu, W. Shen, S. Dong, and M. Chen, "Experimental investigation of fire extinguishment using expansion foam in the underground goaf," Arabian Journal of Geosciences, vol. 8, no. 11, pp. 9055-9063, 2015.

[17] Y. Lu and B. Qin, "Mechanical properties of inorganic solidified foam for mining rock fracture filling," Materials Express, vol. 5, no. 4, pp. 291-299, 2015.

[18] Y. Lu, B. Qin, Y. Jia, Q. Shi, and F. Li, “Thermal insulation and setting property of inorganic solidified foam," Advances in Cement Research, vol. 27, no. 6, pp. 352-363, 2015.

[19] G. Gustafson and H. Stille, "Prediction of groutability from grout properties and hydrogeological data," Tunnelling and Underground Space Technology, vol. 11, no. 3, pp. 325-332, 1996.

[20] T. Fujita, F. Shinkai, and J. Nobuto, "Fundamental study on a grout penetration model for a HLW repository," Journal of Energy and Power Engineering, vol. 6, pp. 1191-1203, 2012.

[21] Z. Yang, K. Hou, and T. Guo, "Study on the effects of different water-cement ratios on the flow pattern properties of cement grouts," Applied Mechanics and Materials, vol. 71-78, pp. 12641267, 2011.

[22] G. Shi, M. Liu, J. Kong, and D. Wang, "Flow characteristics of three-phase foam in mine gob and its application," International Journal of Mining Science and Technology, vol. 25, no. 5, pp. 773778, 2015.

[23] L. Korat and V. Ducman, "The influence of the stabilizing agent SDS on porosity development in alkali-activated fly-ash based foams," Cement and Concrete Composites, vol. 80, pp. 168-174, 2017.

[24] D. Lootens and D. P. Bentz, "On the relation of setting and earlyage strength development to porosity and hydration in cementbased materials," Cement and Concrete Composites, vol. 68, pp. 9-14, 2016.

[25] S. Jacobsen, L. Haugan, T. A. Hammer, and E. Kalogiannidis, "Flow conditions of fresh mortar and concrete in different pipes," Cement and Concrete Research, vol. 39, no. 11, pp. 9971006, 2009.

[26] T. Hirochi, Y. Maeda, S. Yamada, M. Shirakashi, M. Hattori, and A. Saito, "Flow patterns of ice/water slurry in horizontal pipes," Journal of Fluids Engineering, vol. 126, pp. 935-940, 2004.

[27] M.-H. Wang, C. Huang, K. Nandakumar, P. Minev, J. Luo, and S. Chiovelli, "Computational fluid dynamics modelling and experimental study of erosion in slurry jet flows," International Journal of Computational Fluid Dynamics, vol. 23, no. 2, pp. 155172, 2009.

[28] S.-J. Li, L.-G. Wang, Y.-L. Lu, and B. Zhang, "Slurry diffusion within cracked wall rock during the bolt-grouting process," Zhongguo Kuangye Daxue Xuebao/Journal of China University of Mining and Technology, vol. 40, pp. 874-880, 2011.
[29] Y. Li, Y. Shen, and X. Huang, "Test study on diffusion laws of foundation grouting in submarine immersed tube tunnel," China Journal of Underground Space Technology, vol. 8, pp. 1244-1249, 2012.

[30] X. Z. Yang, X. H. Wang, and J. S. Lei, "Study on grouting diffusion radius of Bingham fluids," Journal of Hydraulic Engineering, vol. 6, pp. 75-79, 2004.

[31] D. Wang and W. Sui, "Grout diffusion characteristics during chemical grouting in a deep water-bearing sand layer," International Journal of Mining Science and Technology, vol. 22, no. 4, pp. 589-593, 2012.

[32] D. V. Georgievskii, "Stability of Bingham flows: from the earliest works of A. A. Il'yushin to the present," Journal of Engineering Mathematics, vol. 78, pp. 9-17, 2013.

[33] Z. Q. Yang, K. P. Hou, T. T. Guo, and M. A. Qiu, "Study of column-hemispherical penetration grouting mechanism based on bingham fluid of time-dependent behavior of viscosity," Rock \& Soil Mechanics, vol. 32, pp. 2697-2703, 2011. 

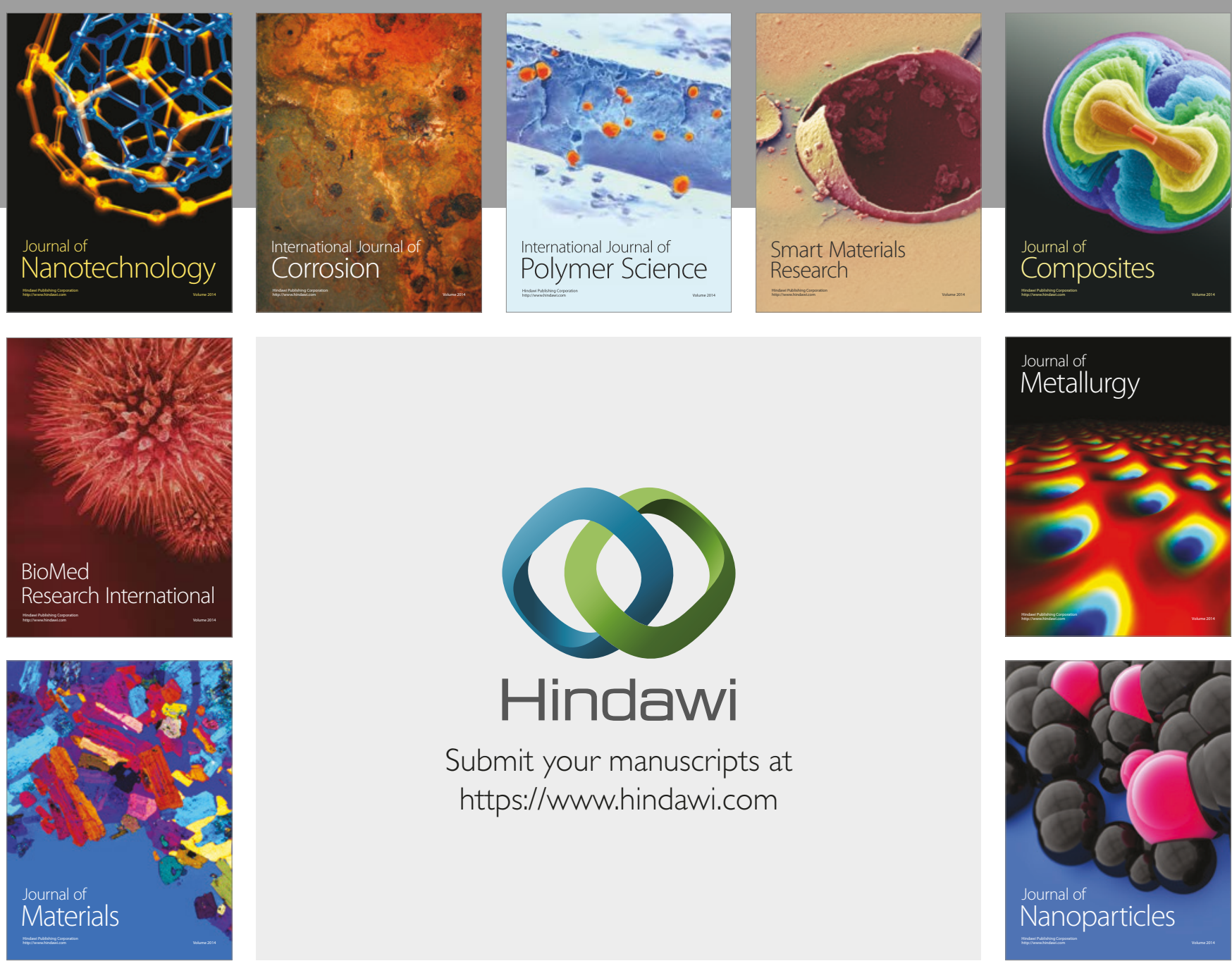

\section{Hindawi}

Submit your manuscripts at

https://www.hindawi.com
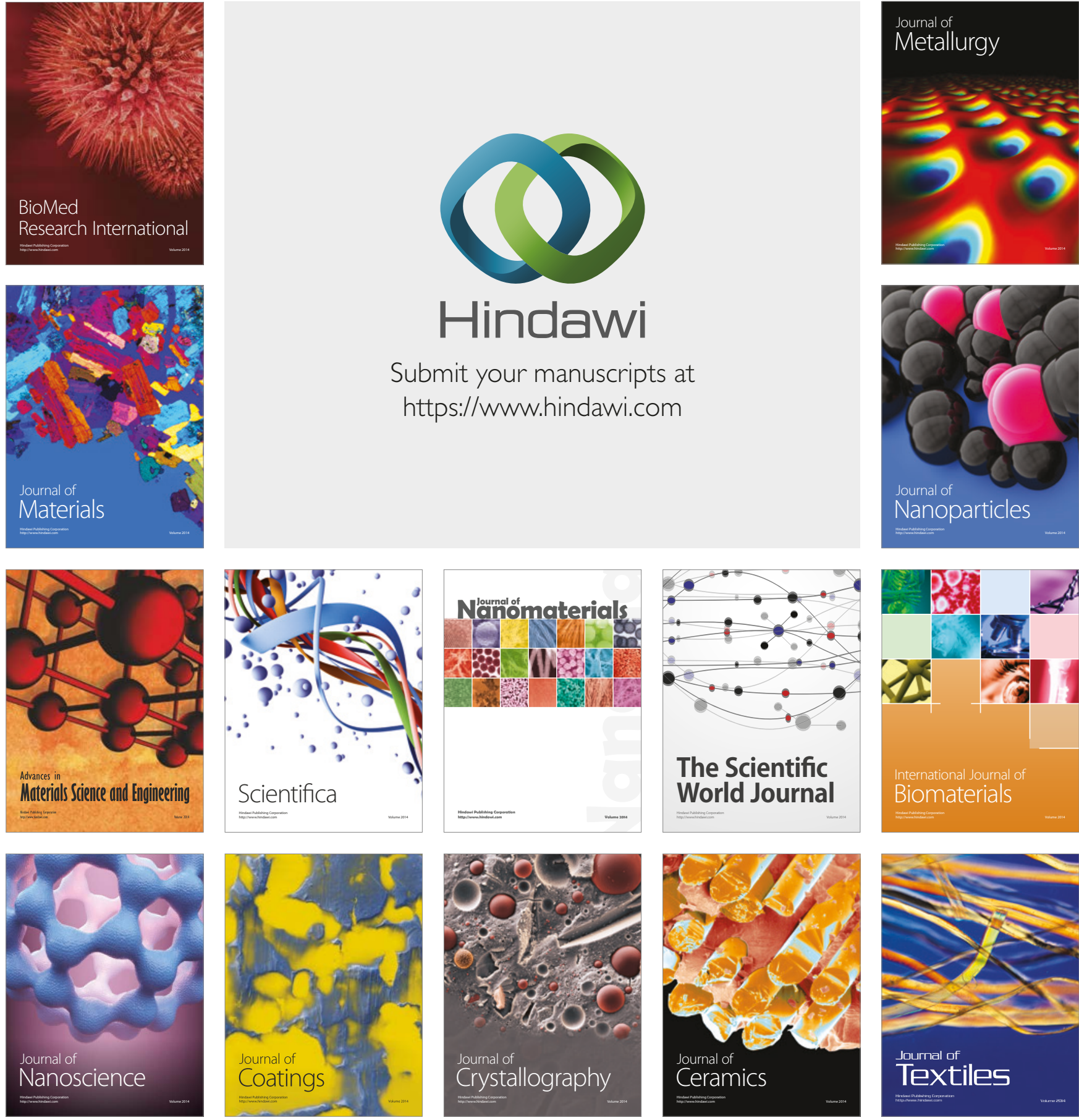

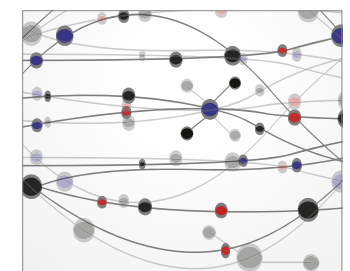

The Scientific World Journal
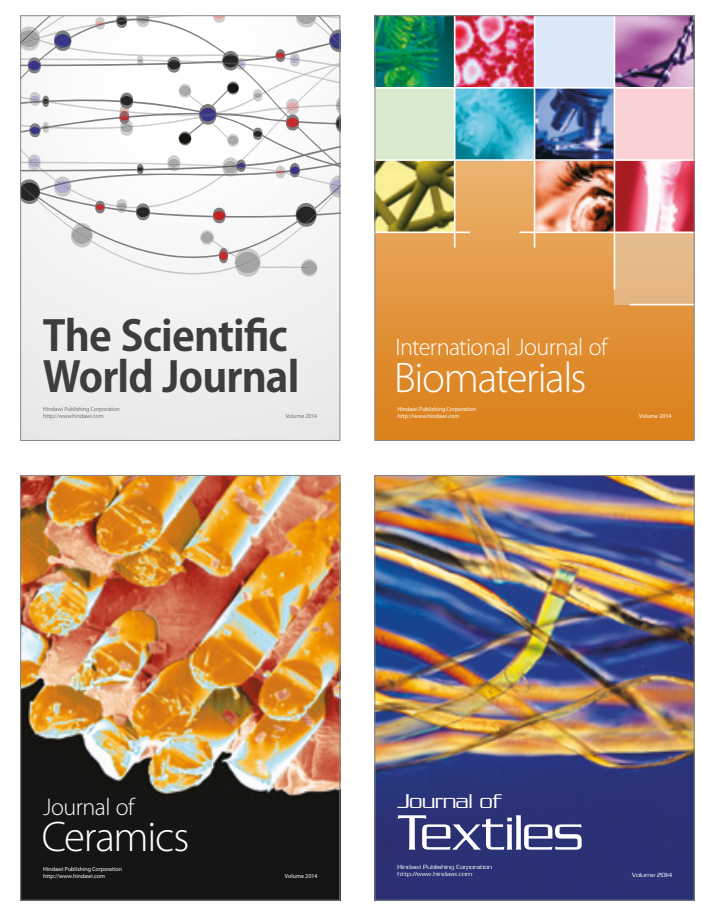